\title{
Morphologie et abondance des stades de développement d'llyocoris cimicoïdes Linné 1758 (Heteroptera : Naucoridae) dans un lac anthropisé en zone tropicale (Cameroun)
}

\author{
Ajeagah Gideon A*.(1), Kekeunou Sévilor(2), Njiawouo Pountigni E. N.(1), Foto Menbohan S.(1) \\ 1. Laboratoire de Biologie Générale, Université de Yaoundé I, B.P. 812 Faculté des Sciences, Cameroun. \\ 2. Laboratoire de Zoologie, Faculté des Sciences, Université de Yaoundé I, B.P. 812, Cameroun. \\ ${ }^{*}$ Correspondance à : ajeagahg@yahoo.com
}

Original submitted in on $6^{\text {th }}$ March 2014. Published online at www.m.elewa.org on $31^{\text {st }}$ July 2014. http://dx.doi.org/10.4314/jab.v79i1.7

\section{RÉSUMÉ}

Objectifs : Une étude conduite au laboratoire de Zoologie et de Biologie Générale de l'Université de Yaoundé I a porté sur l'écologie de la punaise aquatique llyocoris cimicoïdes Linné 1758 (Heteroptera : Naucoridae) prélevée dans le lac d'Obili (Yaoundé, Cameroun).

Méthodologie et résultats : L'analyse physico-chimique montre que ce lac est dans un état eutrophe à hypereutrophe et ses eaux sont fortement chargées en matière organique. Les observations morphologiques et l'illustration des stades de développement ainsi que l'étude de leurs fluctuations numériques montrent que $I$. cimicoïdes à six stades post-embryonnaires parmi lesquels cinq sont larvaires. La taille des individus augmente de la larve de 1 er stade jusqu'au stade adulte. Les stades larvaires d'I. cimicoïdes se distinguent les uns des autres par la présence ou l'absence du ptérothèque, la forme de la marge postérieure du mésotergum et la taille relative du ptérothèque par rapport à la base du mésotergum.

Conclusions : L'abondance des stades de développement d'I.cimicoïdes est influencée par la saisonnalité et l'évolution de certaines variables physico-chimiques. Les larves pullulent pendant la saison sèche et les œufs et adultes pendant la saison des pluies.

Mots clés : Pollution aquatique, morphologie, développement, llyocoris cimicoïdes.

\section{Abstract}

Objectives : A study conducted at the Laboratory of Zoology and General Biology, University of Yaoundé I focused on the ecology of aquatic bug llyocoris cimicoides Linnaeus 1758 (Heteroptera : Naucoridae) taken from Lake Obili (Yaoundé, Cameroon).

Methodology and Results: The physico-chemical analysis showed that this lake is in a eutrophic state and its waters are heavily loaded with organic matter. Morphological observations and illustration stages of development and the study of their digital fluctuations show that $I$. cimicoides, has six post-embryonic stages of which five are breeding. The sizes of individuals increase the first stage larva to adult. Larval stages of $l$. 


\section{Ajeagah et al. J. Appl. Biosci. 2014. Morphologie et abondance des stades de développement}

d'llyocoris cimicoïdes Linne 1758 dans un lac anthropisé en zone tropicale (Cameroun)

cimicoides are distinguished from each other by the presence or absence of pterotheque, the shape of the posterior margin and mesotergum pterotheque relative size with respect to the base of mesotergum.

Conclusions : Abundance stages of development $I$. cimicoides is influenced by seasonality and the evolution of certain physico-chemical variables. Larvae swim in the dry season and eggs and adults during the rainy season.

Keywords : Water Pollution, morphology, development, llyocoris cimicoides,

\section{INTRODUCTION}

Les Naucoridés sont des Hexapodes des eaux stagnantes biens végétalisées. Ils vivent dans les mares, les étangs, les marais et certains ruisseaux à courant lent où ils se localisent parmi les plantes (Dethier, 1980). Ils nagent sur le dos grâce aux soies qui garnissent leurs pattes postérieures bien que celles-ci ne soient pas modifiées en palettes natatoires. Ils bordent la surface de l'eau par l'extrémité dorsale de leur abdomen. Ce sont des prédateurs féroces qui attaquent tous les petits animaux aquatiques en rapport avec leur taille (têtards, larves d'insectes). Leurs aptitudes prédatrices les rend potentiellement aptes à la lutte contre les larves de moustiques (Mohammad et al, 2011 ; Polhemus et Polhemus, 2008). Cependant, la prolifération des Naucoridés représente une menace émergente pour la santé publique dans de nombreuses régions intertropicales humides puisqu'ils seraient impliqués dans la transmission de l'ulcère de Buruli (infection à Mycobacterium ulcerans) à l'Homme (OMS, 2004 ; Aubry, 2011). Leur comportement migratoire favorise la dissémination de $M$. ulcerans, ainsi que sa transmission, hors de l'environnement aquatique (Gamboa et al, 2012 ; Ebong et al, 2012). La famille des Naucoridae est représentée par environ 36

\section{MATÉRIEL ET MÉTHODES}

Site d'étude : Cette étude a été menée dans un lac du quartier Obili à Yaoundé (Cameroun). Yaoundé est une ville située dans la région écologique du Sud forestier camerounais (qui s'étend entre $3^{\circ} 30^{\prime}$ et $3^{\circ} 58^{\prime}$ de latitude Nord et entre $11^{\circ} 20^{\prime}$ et $11^{\circ} 40^{\prime}$ de longitude Est). Le site d'étude est sous la dépendance d'un climat équatoriale classique à quatre saisons d'importance et de durées inégales : une grande saison sèche (de mi-novembre à mi-mars) ; une petite saison des pluies (de mi-mars à fin juin) ; une petite saison sèche (de juillet à août) et une grande saison des pluies (de septembre à mi-novembre). De forme ovoïde irrégulière (Figure 1), le lac d'Obili (351' genres connus, avec environ 370 espèces décrites (Sites et al, 2011). Parmi les représentants camerounais de cette famille, le genre Ilyocoris regroupe les Naucoridae qui ont les bords latéraux de leur pronotum lisses et nettement rebordés (Tachet et al, 2002). Au Cameroun, les informations scientifiques sont rares sur llyocoris cimicoïdes. Sa morphométrie, sa morphologie et sa systématique restent très peu documentées et illustrés dans son aire de répartition. Cependant, les adultes de cette espèce ont une longueur moyenne de $15 \mathrm{~mm}$. Ils ont un corps vert olivâtre, une tête, un pronotum et un scutellum brillants. Les tibias intermédiaires et postérieurs sont hérissés de fortes épines (Poisson, 1948). Ces connaissances, encore fragmentaires et incomplètes rendent difficiles une meilleur connaissance de ce cette espèce. La distinction des différents stades de développement d'I.cimicoïdes est difficile. Les objectifs de ce travail sont: (i) la détermination des caractéristiques morphologiques des différents stades de développement d'l. cimicoïdes; (ii) l'établissement d'une clé d'identification des différents stades larvaires d' $l$. cimicoïdes et (iii) la détermination des périodes de pullulation d'I.cimicoïdes dans son habitat naturel.

de latitude Nord, $11^{\circ} 29^{\prime}$ de longitude Est) est situé à 720 $\mathrm{m}$ de hauteur ; il mesure environ $100 \mathrm{~m}$ de long et $60 \mathrm{~m}$ de large pour une colonne d'eau d'environ $2,5 \mathrm{~m}$ de profondeur maximale. Il est utilisé à des fins piscicoles et borde du coté de sa rive gauche de petits étangs artificiels servant de site de multiplication des alevins du MINEPIA (Ministère de l'Élevage, de la Pèche et des Industries Animales).

Analyses physico-chimiques : Les échantillonnages ont été effectués de décembre 2011 à juillet 2012 au niveau de la zone littorale du lac à raison de deux échantillons par mois. Ces échantillons d'eau destinés 


\section{Ajeagah et al. J. Appl. Biosci. 2014. Morphologie et abondance des stades de développement}

d'llyocoris cimicoïdes Linne 1758 dans un lac anthropisé en zone tropicale (Cameroun)

aux analyses physico-chimiques ont été prélevés en surface et en profondeur (à $0,3 \mathrm{~m}$ environ) et conservés dans des flacons en polyéthylène à double bouchage de $1000 \mathrm{ml}$. Les analyses physico-chimiques, faites suivant une méthodologie appropriée (Rodier, 1996; APHA, 1998), ont portées sur la température, le potentiel d'Hydrogène $(\mathrm{pH})$, la transparence, la couleur, la turbidité, les teneurs en gaz carbonique dissous, en oxygène dissous, en composés azotés et en orthophosphates, la Demande Biochimique en Oxygène $\left(\mathrm{DBO}_{5}\right)$, la conductivité électrique, l'alcalinité et la dureté calcique.

Échantillonnage des Naucoridés : Les échantillons de Naucoridae ont été récoltés dans la zone littorale du lac une fois par mois grâce à un filet troubleau de forme carrée et suivant l'approche multi habitat (Barbour et al, 1999 ; Moisan et Pelletier, 2008). La coloration de chaque individu collecté a été notée et les spécimens ont été conservés dans des piluliers contenant une solution de formol à $10 \%$.

Identification d'llyocoris cimicoïdes et dénombrement des stades de développement: Au laboratoire, les Naucoridae récoltés ont été nettoyés à l'eau courante et grâce aux clés de Poisson (1957) et de Sites et Nichols (2001) les individus appartenant à l'espèce I. cimicoïdes ont été identifiés. Après avoir dénombré les œufs et les individus de différents stades de développement postembryonnaire, ces spécimens ont été conservés dans des piluliers étiquetés contenant de l'alcool à $70 \%$.

Étude morphométrique et morphologique d'llyocoris cimicoïdes : Les mensurations ont été effectuées sur 130 individus de chacun des stades de développement au cours des observations à la loupe binoculaire de marque WILD $M_{5}$ munie d'un micromètre oculaire. Les mensurations de l'œuf ont porté sur sa longueur et sa largeur (seule la distance séparant les extrémités antérieures et postérieures est prise en compte pour la longueur tandis que la largeur est mesurée dans la zone médiane). Les mensurations effectuées sur les larves ont porté sur la longueur et la largeur du corps ; la longueur et la largeur de la tête; la longueur des différents sclérites thoraciques (à savoir le pronotum, le mésonotum et le métanotum) et la longueur des articles des différentes pattes. La longueur du corps est mesurée entre les extrémités antérieure et postérieure .alors que la largeur du corps est mesurée dans la zone médiane la plus large, sur la face dorsale. Les mesures effectuées sur les adultes ont porté sur la largeur du synthlipsis (espace interoculaire), la longueur et la largeur de l'aile antérieure ainsi que les organes mesurés chez les larves Pour faciliter les observations morphologiques, la chitine a été préalablement ramollie et éclaircie en plongeant les spécimens à étudier dans une solution de soude caustique à $10 \%$ dans laquelle ils ont séjourné le temps nécessaire pour leur ramollissement et leur éclaircissement. Chaque spécimen a été ensuite larvé et rincé dans trois bains successifs d'alcool à $70 \%$. Les individus éclaircis de chaque stade de développement post-embryonnaire ainsi que les œufs ont été convenablement montés entre lame et lamelle et examinés à la loupe binoculaire de marque WILD M-97339 $_{\text {- }}$ muni d'un tube à dessin qui a permis la réalisation des dessins.

Analyse des données: Les logiciels Microsoft Office Excel 2007 et SPSS 17.0 nous ont permis :

(i) d'établir la statistique descriptive des individus de différents stades de développement;

(ii) d'évaluer l'abondance relative $\left(P_{i}=n_{j} / N\right)$ des individus de différents stades $\left(n_{i}\right)$ présent par rapport au nombre total d'individus $(\mathrm{N})$;

(iii) de calculer le coefficient de corrélation "r" de Spearman entre l'abondance des différents stades de développement d'llyocoris cimicoïdes et les variables physico-chimiques. 


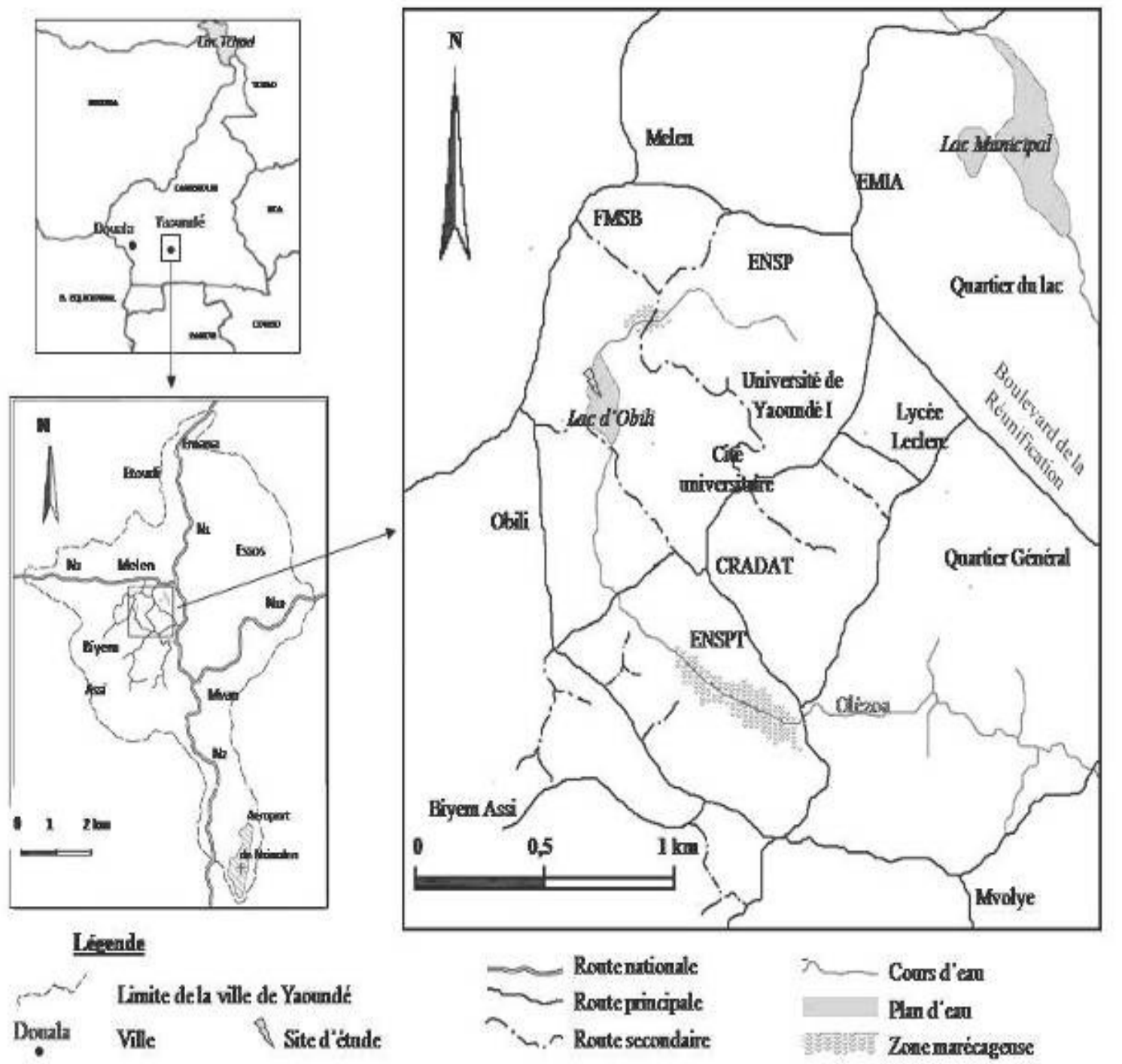

Figure 1 : Présentation du site d'étude dans la ville de Yaoundé.

\section{RÉSULTATS}

Evolution temporelle des variables physicochimiques du lac d'Obili (Yaoundé, Cameroun): Durant la période d'étude, le pH de l'eau a varié de 5,12à 7,26 UC et la transparence de 0,5 à $0,67 \mathrm{~m}$. Les températures ont varié de 25 à $32^{\circ} \mathrm{C}$ en surface et de 23 à $28^{\circ} \mathrm{C}$ en profondeur, la conductivité électrique de 127,3 à $240 \mu \mathrm{S} / \mathrm{cm}$ en surface et de 124 à $255 \mu \mathrm{S} / \mathrm{cm}$ en profondeur. Les eaux de surface avec une turbidité variant de 16 à 56 FTU ont été moins troubles que celles des profondeurs où celle-ci ont fluctué de 35 à 200 FTU. La couleur a varié entre 78 et 298 Pt-Co. Les teneurs en $\mathrm{CO}_{2}$ dissous ont varié de 2 à $15,84 \mathrm{mg} / \mathrm{L}$ en surface et de 0,2 à $19,36 \mathrm{mg} / \mathrm{L}$ en profondeur alors que les teneurs en oxygène dissous ont été comprises respectivement entre 5,2 et $8,56 \mathrm{mg} / \mathrm{L}$ et entre 0,83 et $7,82 \mathrm{mg} / \mathrm{L}$ en surface et en profondeur. Les valeurs de la $\mathrm{DBO}_{5}$ ont variés de 40 à $140 \mathrm{mg} / \mathrm{L}$ en surface tandis qu'en profondeur, elles ont varié de 30 à $85 \mathrm{mg} / \mathrm{L}$. Les teneurs en nitrites ont varié de 0,02 et $0,06 \mathrm{mg} / \mathrm{L}$ en surface et de 0,016 et $0,4 \mathrm{mg} / \mathrm{L}$ en profondeur. Les taux de nitrates enregistrés ont oscillé entre 0,4 et $10 \mathrm{mg} / \mathrm{L}$ en surface et entre 0,1 et 20,2 mg/L en profondeur alors que 
les concentrations en azote ammoniacal dans l'ensemble ont varié de 0,16 à $33,28 \mathrm{mg} / \mathrm{L}$. Les relevés de la teneur de l'eau en orthophosphates ont présenté en surface des valeurs comprises entre 0,7 et $1,63 \mathrm{mg} / \mathrm{L}$ et en profondeur des valeurs comprises entre 0,44 et 1,79 $\mathrm{mg} / \mathrm{L}$. Les valeurs de l'alcalinité en surface et en profondeur ont respectivement varié de 4,3 à $200 \mathrm{mg} / \mathrm{L}$ et de 3,7 à $132 \mathrm{mg} / \mathrm{L}$. Les valeurs de la dureté calcique ont été comprises entre 0,2 et $78,5 \mathrm{mg} / \mathrm{L}$. La teneur moyenne en orthophosphates a été de 1,1 $\pm 0,01 \mathrm{mg} / \mathrm{L}$ et la transparence moyenne de 0,58 $\pm 0,2 \mathrm{~m}$. Conformément aux critères de classification du statut trophique des plans d'eau, ces valeurs permettent de classer le lac d'Obili dans la catégorie des milieux eutrophes à hypereutrophes.
Analyse des corrélations entre les variables physicochimiques du lac et l'abondance des stades de développement d'l. cimicoïdes: Les valeurs des coefficients de corrélations linéaires entre les variables physico-chimiques et l'abondance des stades de développement d'l. cimicoïdes n'ont pas été significatives (Tableau 1). Cependant, l'abondance des larves de 3ème stade a été significativement et positivement corrélée à la couleur mais négativement corrélée à la turbidité. L'abondance des larves de $4^{\mathrm{ème}}$ stade a été significativement et négativement corrélée à la turbidité et aux nitrates. Celle des larves de 5 ème stade a été significativement et positivement corrélée au $\mathrm{pH}$ mais négativement corrélée à la turbidité (Tableau 1).

Tableau 1 : Valeurs des coefficients de Corrélations linéaires entre les paramètres physico-chimiques de l'eau du lac d'Obili (Yaoundé, Cameroun) et l'abondance des stades de développent d'l. cimicoïdes

\begin{tabular}{|c|c|c|c|c|c|c|c|c|c|c|c|c|c|c|}
\hline & Temp. & $\mathbf{p H}$ & $\mathrm{CO}_{2}$ & $\mathrm{O}_{2}$ & $\mathrm{DBO}_{5}$ & Cond & $\begin{array}{l}\text { Cor } \\
\text { lexi }\end{array}$ & $\begin{array}{c}\text { Tubi } \\
\text { dité }\end{array}$ & Ninite & Ninte & $\begin{array}{l}\text { Azste } \\
\text { Ammo. }\end{array}$ & $\begin{array}{l}\text { Oftho } \\
\text { Foos } \\
\text { phate }\end{array}$ & $\begin{array}{c}\text { Albi } \\
\text { nité }\end{array}$ & $\begin{array}{l}\text { Duté } \\
\text { clicique }\end{array}$ \\
\hline Eufs & $-0,331$ & $-0,095$ & 0,252 & 0,048 & 0,299 & 0,119 & $-0,429$ & $-0,024$ & $-0,167$ & $-0,119$ & 0,024 & $-0,132$ & 0,333 & 0,310 \\
\hline Lavede stade1 & 0,307 & $-0,690$ & $-0,204$ & 0,143 & 0,443 & 0,143 & 0,490 & 0,000 & $-0,452$ & $-0,643$ & 0,262 & $-0,491$ & 0,500 & $-0,548$ \\
\hline Lave de tade 2 & 0,500 & 0,335 & 0,271 & 0,036 & 0,495 & $-0,287$ & 0,515 & 0,599 & 0,311 & $-0,599$ & 0,036 & 0,313 & 0,371 & 0,563 \\
\hline Lave de stade 3 & 0,395 & 0,539 & 0,494 & 0,503 & 0,422 & $-0,084$ & $0,790^{*}$ & $-0,755^{*}$ & 0,671 & $-0,048$ & 0,383 & 0,060 & 0,275 & $-0,431$ \\
\hline Lave de stade 4 & 0,543 & 0,216 & 0,253 & $-0,275$ & 0,530 & $-0,156$ & 0,551 & $0,755^{*}$ & 0,444 & $-0,802^{*}$ & 0,431 & 0,120 & 0,252 & $-0,599$ \\
\hline Lave de tades & $-0,184$ & $0,714^{*}$ & 0,671 & 0,643 & 0,180 & 0,405 & 0,000 & $0,714^{*}$ & 0,500 & $-0,143$ & $-0,810^{*}$ & 0,683 & 0,333 & 0,190 \\
\hline Adille & $-0,342$ & $-0,048$ & 0,279 & $-0,133$ & 0,212 & 0,084 & $-0,325$ & $-0,120$ & $-0,145$ & $-0,072$ & 0,241 & $-0,121$ & 0,386 & 0,289 \\
\hline
\end{tabular}

* = conáation significative an seuil $5 \%$

Caractéristiques morphologiques des différents stades de développement d'l. cimicoïdes : OEuf : De forme ovalaire, l'œuf d'llyocoris cimicoïdes est vert et marron à l'extrémité antérieure. L'œuf mûr montre par transparence dans sa région antérieure les deux yeux de la future larve (Figure 2). La longueur de l'œuf varie de 1,4 à 2,52 $\mathrm{mm}$ (en moyenne 1,84 $\pm 0,018 \mathrm{~mm}$ ) et sa largeur de 0,88 à 1,12 $\mathrm{mm}$ (en moyenne 1,02 $\pm 0,004$ $\mathrm{mm})$. A la ponte, les œufs sont accolés sur le dos d'un adulte 


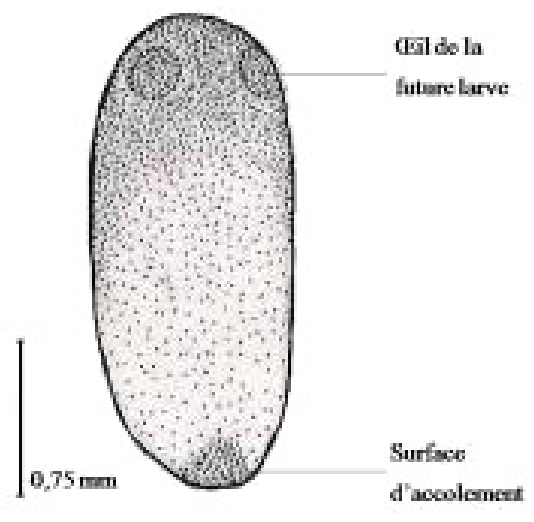

Figure 2 : Morphologie de l'œuf d'llyocoris cimicoïdes

Stades larvaires : Les larves sont brunes verdâtres. La couleur des yeux varie, elle est noire sur la face dorsale et rouge sur la face ventrale. L'extrémité du tarse des pattes antérieures est rouge orangée alors que les tarses moyens et postérieurs sont bruns. Le connexivum (bords latéraux des segments abdominaux, région pleurale) est brunâtre. Elles sont aplaties dorsoventralement. Le pronotum à une forme trapézoïdale et sa marge antérieure est concave alors que la marge postérieure présente une légère convexité. Les tibias moyens et postérieurs portent plusieurs rangées d'épines. L'abdomen, visiblement segmenté, compte 8 segments et revêtu ventralement d'une pilosité hydrofuge. La larve de premier stade est caractérisée par une longueur variant de 3,4 à 4,28 $\mathrm{mm}$ et une largeur variant de 2,04 à 2,32 $\mathrm{mm}$ (Tableau 2). La longueur de la tête varie de 0,36 à $0,64 \mathrm{~mm}$; sa largeur varie de 1,16 à $1,36 \mathrm{~mm}$. La longueur du pronotum est de 0,36 à 0,56 ; celle du mésonotum et du métanotum sont respectivement de 0,32 à 0,56 et de 0,16 à 0,32 . Le profémur mesure 0,92 à $1,24 \mathrm{~mm}$ de longueur celle du protibia est de 0,56 à 0,76 ; celle du protarse varie de 0,20 à 0,36 ; celle du mésofémur est de 0,92 à 1,16 ; celle du mésotibia est de 0,76 à 1,08 ; celle du mésotarse varie de 0,32 à 0,56 ; celle du métafémur varie de 1,04 à 1,28 ; celle du métatibia est de 1,12 à 1,32; celle du métatarse varie de 0,64 à 0,84 . Cette larve est environ 1,7 fois plus longue que large, la tête est 2,5 fois plus large que longue. Le pronotum et le mésenotum sont de taille presqu'égale, mais environ 1,7 fois la taille $d u$ métanotum qui est le plus petit segment thoracique. Le ptérothèque est absent (Figure 4A). La larve de deuxième stade mesure 4,72 à $5,36 \mathrm{~mm}$ de long et 2,72 à 3,20 de large (Tableau 2). La longueur de la tête varie de 0,56 à 0,88 ; une largeur variant de 1,52 à 1,72. La longueur du pronotum est de 0,48 à 0,68 ; celle du mésonotum et du métanotum sont respectivement de 0,60 à 0,76 et de 0,20 à 0,40 . Le profémur mesure 1,24 à $1,48 \mathrm{~mm}$ de longueur ; celle du protibia est de 0,76 à 0,92 ; celle du protarse varie de 0,28 à 0,36 ; celle du mésofémur est de 1,20 à 1,56 ; celle du mésotibia est de 1,00 à 1,16 ; celle du mésotarse varie de 0,52 à 0,64 ; celle du métafémur varie de 1,48 à 1,68; celle du métatibia est de 1,48 à 1,64 ; celle du métatarse varie de 0,72 à 0,88 . Cette larve est environ 1,6 fois plus longue que large, sa tête est toujours 2,5 fois plus large que longue. Le pronotum est légèrement plus petit que le mésonotum et le métanotum demeure le plus petit segment thoracique (Figure 4B). Le bord médian du mésotergum présente une légère échancrure. Le ptérothèque est présent ; il est d'allure triangulaire et son extrémité atteint la base du mésotergum pris au niveau de la ligne médiane. 


\section{Ajeagah et al. J. Appl. Biosci. 2014. Morphologie et abondance des stades de développement}

d'llyocoris cimicoïdes Linne 1758 dans un lac anthropisé en zone tropicale (Cameroun)

Tableau 2 : Dimensions ( $\mathrm{mm}$ ) des différentes parties corporelles des larves et adultes d'llyocoris cimicoïdes prélevées dans un lac Eutrophe d'Obili (Yaoundé, Cameroun) de décembre 2011 à juillet 2012

\begin{tabular}{|l|c|c|c|c|c|c|}
\hline & $\begin{array}{c}\text { Larve de } \\
\text { stade } \\
\mathbf{1 ( n = 1 0 0 )}\end{array}$ & $\begin{array}{c}\text { Larve de } \\
\text { stade } \\
\mathbf{2 ( n = 1 0 0 )}\end{array}$ & $\begin{array}{c}\text { Larve de } \\
\text { stade } \\
\mathbf{3 ( n = 1 0 0 )}\end{array}$ & $\begin{array}{c}\text { Larve de } \\
\text { stade } \\
\mathbf{4}(\mathbf{n}=100)\end{array}$ & $\begin{array}{c}\text { Larve de } \\
\text { stade } \\
\mathbf{5}(\mathbf{n}=100)\end{array}$ & $\begin{array}{c}\text { Adulte } \\
(\mathbf{n}=130)\end{array}$ \\
\hline Longueur du corps & $3,90 \pm 0,009$ & $4,96 \pm 0,009$ & $6,69 \pm 0,011$ & $8,75 \pm 0,025$ & $11,31 \pm 0,031$ & $14,93 \pm 0,033$ \\
\hline Largeur du corps & $2,18 \pm 0,005$ & $3,03 \pm 0,006$ & $3,96 \pm 0,009$ & $5,21 \pm 0,013$ & $6,81 \pm 0,017$ & $9,75 \pm 0,024$ \\
\hline Longueur de la tête & $0,51 \pm 0,004$ & $0,65 \pm 0,004$ & $0,85 \pm 0,005$ & $1,06 \pm 0,006$ & $1,26 \pm 0,009$ & $1,60 \pm 0,018$ \\
\hline Largeur de la tête & $1,29 \pm 0,002$ & $1,65 \pm 0,002$ & $2,08 \pm 0,0040$ & $2,53 \pm 0,006$ & $3,27 \pm 0,009$ & $4,01 \pm 0,009$ \\
\hline Longueur pronotum & $0,43 \pm 0,003$ & $0,58 \pm 0,003$ & $0,81 \pm 0,003$ & $1,08 \pm 0,005$ & $1,37 \pm 0,007$ & $2,23 \pm 0,010$ \\
\hline Longueur mésonotum & $0,44 \pm 0,003$ & $0,66 \pm 0,002$ & $0,98 \pm 0,004$ & $1,41 \pm 0,006$ & $2,18 \pm 0,010$ & $3,04 \pm 0,013$ \\
\hline Longueur métanotum & $0,25 \pm 0,002$ & $0,31 \pm 0,002$ & $0,43 \pm 0,002$ & $0,49 \pm 0,003$ & $0,56 \pm 0,005$ & $0,62 \pm 0,005$ \\
\hline Profémur & $1,09 \pm 0,004$ & $1,34 \pm 0,003$ & $1,69 \pm 0,003$ & $2,02 \pm 0,005$ & $2,53 \pm 0,006$ & $3,20 \pm 0,007$ \\
\hline Protibia & $0,66 \pm 0,003$ & $0,84 \pm 0,002$ & $1,02 \pm 0,003$ & $1,19 \pm 0,005$ & $1,51 \pm 0,005$ & $1,91 \pm 0,006$ \\
\hline Protarse & $0,30 \pm 0,002$ & $0,34 \pm 0,001$ & $0,41 \pm 0,001$ & $0,45 \pm 0,003$ & $0,54 \pm 0,004$ & $0,64 \pm 0,003$ \\
\hline Mésofémur & $1,04 \pm 0,003$ & $1,33 \pm 0,004$ & $1,74 \pm 0,004$ & $2,18 \pm 0,006$ & $2,84 \pm 0,008$ & $3,60 \pm 0,011$ \\
\hline Mésotibia & $0,85 \pm 0,004$ & $1,10 \pm 0,002$ & $1,42 \pm 0,003$ & $1,74 \pm 0,005$ & $2,23 \pm 0,007$ & $2,89 \pm 0,010$ \\
\hline Mésotarse & $0,48 \pm 0,002$ & $0,58 \pm 0,002$ & $0,70 \pm 0,002$ & $0,84 \pm 0,003$ & $1,09 \pm 0,005$ & $1,32 \pm 0,005$ \\
\hline Métafémur & $1,20 \pm 0,003$ & $1,57 \pm 0,002$ & $2,08 \pm 0,003$ & $2,64 \pm 0,005$ & $3,53 \pm 0,010$ & $4,51 \pm 0,010$ \\
\hline Métatibia & $1,20 \pm 0,003$ & $1,56 \pm 0,002$ & $2,03 \pm 0,004$ & $2,56 \pm 0,006$ & $3,39 \pm 0,009$ & $4,41 \pm 0,009$ \\
\hline Métatarse & $0,70 \pm 0,002$ & $0,83 \pm 0,002$ & $1,03 \pm 0,002$ & $1,25 \pm 0,005$ & $1,67 \pm 0,007$ & $2,09 \pm 0,006$ \\
\hline
\end{tabular}

Les valeurs du tableau représentent des moyennes et leurs erreurs standards. $n=$ nombre d'individus mesurés

La larve de troisième stade est caractérisée par une longueur qui varie de 6,32 à 7,04 $\mathrm{mm}$ et une largeur de 3,68 à 4,24 (Tableau 2). La longueur de la tête varie de 0,72 à 0,96 ; une largeur variant de 1,96 à 2,24 . La longueur du pronotum est de 0,68 à 0,92 ; celle du mésonotum et du métanotum sont respectivement de 0,84 à 1,12 et de 0,36 à 0,52 . Le profémur mesure 1,60 à $1,84 \mathrm{~mm}$ de longueur; celle du protibia est de 0,92 à 1,12 ; celle du protarse varie de 0,36 à 0,48 ; celle du mésofemur est de 1,60 à 1,84 ; celle du mésotibia est de 1,28 à 1,56 ; celle du mésotarse varie de 0,64 à 0,76 ; celle du métafemur varie de 1,96 à 2,20 ; celle du métatibia est de 1,88 à 2,22 ; celle du métatarse varie de 0,88 à 1,12 . Le mésonotum de cette larve est 1,2 fois plus grand que le pronotum et 2,2 fois que le métanotum. Le ptérothèque, toujours d'allure triangulaire, est plus développé que celui de la larve de $2^{\text {ème }}$ stade. Son extrémité, orientée latéralement, est plus allongée que la base du mésotergum (Figure 4C). La larve de quatrième stade mesure 8,16 à $9,44 \mathrm{~mm}$ de long et 4,80 à 5,52 de large (Tableau 2). La longueur de la tête varie de 0,88 à 1,12 ; une largeur variant de 2,32 à 2,72 . La longueur du pronotum est de 0,96 à 1,28 ; celle du mésonotum et du métanotum sont respectivement de 1,12 à 1,52 et de 0,40 à 0,56 . Le profémur mesure 1,92 à $2,24 \mathrm{~mm}$ de longueur; celle du protibia est de 1,04 à 1,44; celle du protarse varie de 0,40 à 0,56 ; celle du mésofémur est de
2,00 à 2,32; celle du mésotibia est de 1,60 à 1,92; celle du mésotarse varie de 0,72 à 0,96 ; celle du métafémur varie de 2,48 à 2,80 ; celle du métatibia est de 2,40 à 2,72; celle du métatarse varie de 1,12 à 1,36. Le mésonotum de cette larve est 1,3 fois plus grand que le pronotum et 2,8 fois que le métanotum. La partie postérieure du mésotergum a une forme arrondie et confère au mésonotum une allure triangulaire caractéristique du meséscutellum différencié des individus adultes. Comme dans le cas de la larve de $3^{\text {ème }}$ stade, le ptérothèque est plus allongé que la partie postérieure du mésotergum (Figure 4D). La larve de cinquième stade mesure 10,72 à $12,00 \mathrm{~mm}$ de long et 6,48 à 7,28 de large (Tableau 2). La longueur de la tête varie de 1,12 à 1,52; une largeur variant de 3,04 à 3,52. La longueur du pronotum est de 1,20 à 1,52; celle du mésonotum et du métanotum sont respectivement de 1,92 à 2,32 et de 0,48 à 0,64 . Le profémur mesure 2,40 à $2,72 \mathrm{~mm}$ de longueur; celle du protibia est de 1,36 à 1,68 ; celle du protarse varie de 0,48 à 0,64 ; celle du mésofémur est de 2,64 à 3,04; celle du mésotibia est de 2,08 à 2,40; celle du mésotarse varie de 0,96 à 1,20 ; celle du métafémur varie de 3,28 à 3,76 ; celle du métatibia est de 3,20 à 3,68 ; celle du métatarse varie de 1,52 à 1,84 . Chez la larve de cinquième stade, le mésonotum est 1,6 fois plus grand que le pronotum et 3,8 fois que le métanotum. Le mésoscutellum est plus 


\section{Ajeagah et al. J. Appl. Biosci. 2014. Morphologie et abondance des stades de développement}

d'llyocoris cimicoïdes Linne 1758 dans un lac anthropisé en zone tropicale (Cameroun)

différencié et le ptérothèque est bien développé et son extrémité orientée vers l'extérieur atteint la limite supérieure du premier segment abdominal (Figure $4 \mathrm{E}$ ). Clé dichotomique d'identification des larves d'l. cimicoïdes : Les éléments pour la distinction spécifique des stades larvaires d'l. cimicoïdes sont résumés dans la clé dichotomique suivante :

1. Longueur du corps inférieure à $4,5 \mathrm{~mm}$, ptérothèques absents, pronotum et mésonotum de taille égale Larve de premier stade

- Longueur du corps supérieure à $4,5 \mathrm{~mm}$, ptérothèques présents, pronotum et mésonotum de taille inégale.

2. Mésotergum, non différencié en un mésoscutellum, présentant une marge postérieure médiane légèrement échancrée.
- Mésotergum, différencié en un mésoscutellum, présentant une marge postérieure médiane non échancrée, concave ou légèrement arrondie

3. Extrémité du ptérothèque atteignant la limite postérieure du mésotergum

Larve de deuxième stade

- Extrémité du ptérothèque dépassant la limite postérieure du mésotergum Larve de troisième stade 4. Extrémité du ptérothèque n'atteignant pas la base du métatergum. Larve de quatrième stade - Extrémité du ptérothèque dépassant légèrement la base du métatergum et atteignant le premier segment abdominal visible. Larve de cinquième stade
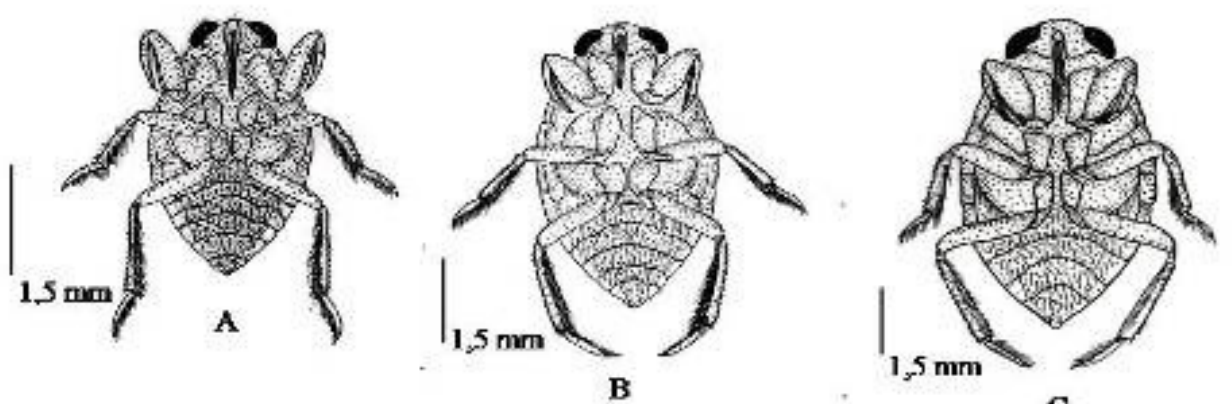

C
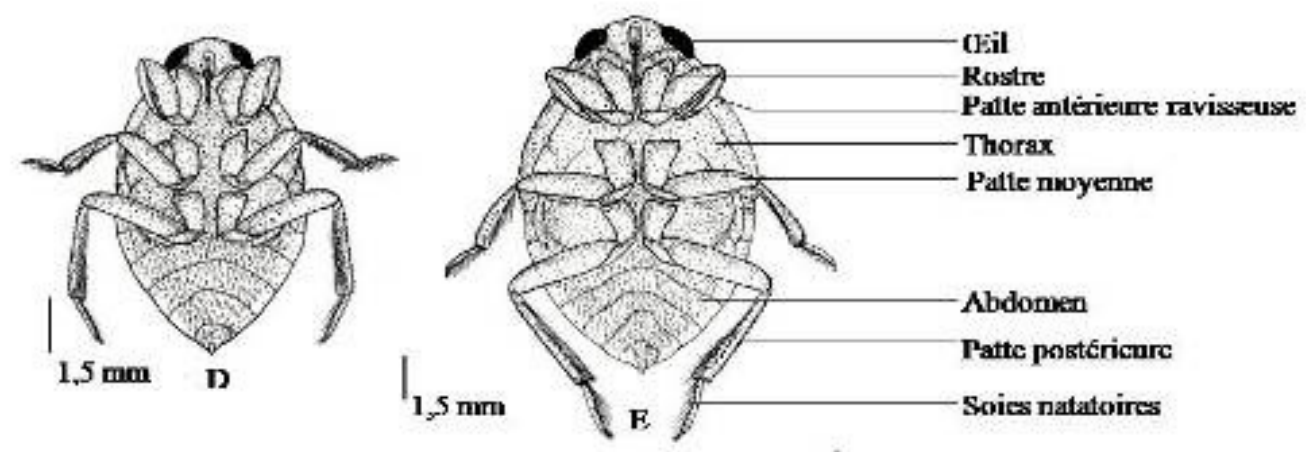

Figure 3: Morphologie des différents stades larvaires d'llyocoris cimicoïdes en vue ventrale A : larve de stade 1, B : larve de stade 2, C : larve de stade 3, D : larve de stade 4, E : larve de stade 5 

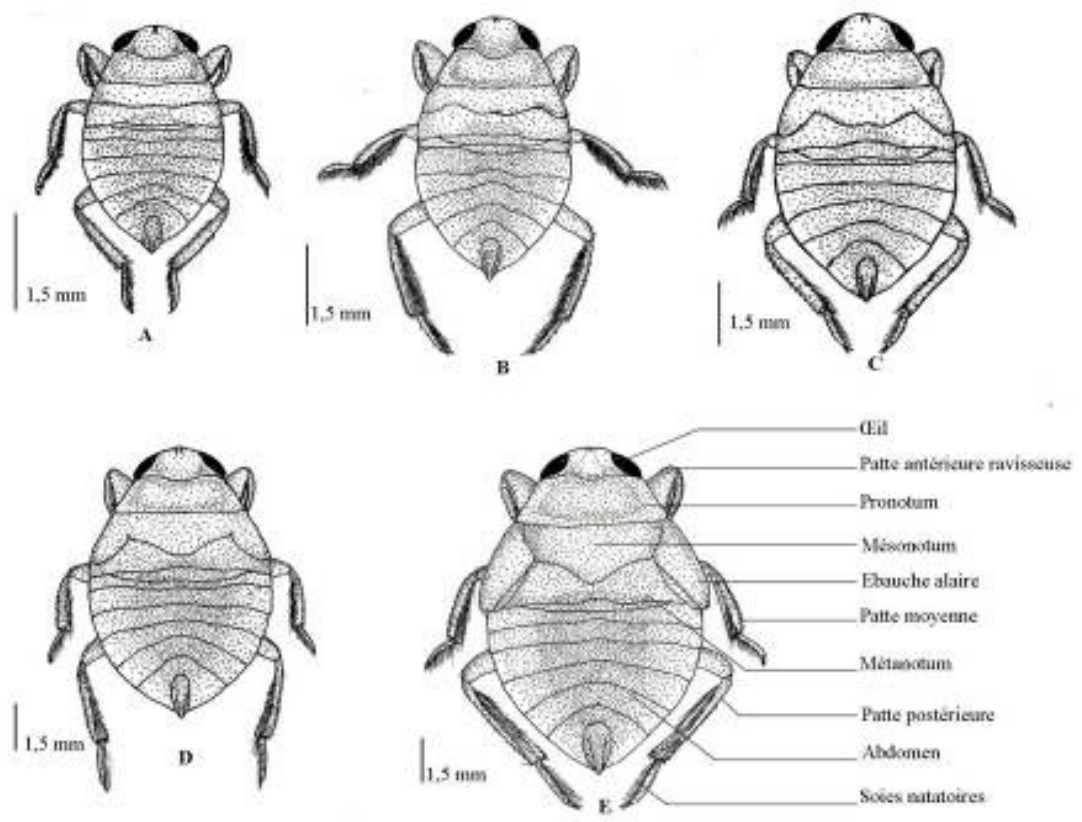

Figure 4 : Morphologie des différents stades larvaires d'llyocoris cimicoïdes en vue dorsale A : larve de stade 1, B : larve de stade 2, C : larve de stade 3, D : larve de stade 4, E : larve de stade 5

Stade adulte : Les individus adultes ont leur face dorsale brun verdâtre. La tête, le pronotoum et le scutullum sont d'un fond brun marron. Les yeux sont noirs et ponctuées de rouge sur la face ventrale. Le rostre est jaunâtre ; le pronotum présente une marge latérale et une large bande basale jaunâtre. Les hémélytres sont d'un brun olivâtre, opaque avec le clavus et la marge plus pâles. Les tibias des pattes méso et métathoraciques sont hérissés d'épines brunâtres. Le connexivum est également brunâtre. La face ventrale de l'abdomen est noirâtre. L'adulte est caractérisé par une longueur qui varie de 14,24 à 15,84 mm et une largeur de 8,00 à $9,28 \mathrm{~mm}$ (Tableau 2). La longueur de la tête varie de 1,28 à 2,24 ; sa largeur de 3,84 à 4,16 et l'espace interoculaire de 1,92 à 2,40 (en moyenne 2,12 $\pm 0,009$ ). La longueur du pronotum est de 2,08 à 2,56 . Le mésoscutellum mesure longitudinalement au niveau de la ligne médiane entre 2,72 à 3,36 (en moyenne $3,04 \pm 0,013$ ). L'hémélytre a une longueur totale variant de 10,4 à 11,84 (en moyenne $11,16 \pm 0,025 \mathrm{~mm}$ ) et une largeur variant de 4,16 à 4,80 (en moyenne de $4,53 \pm 0,013$ ). Le profémur mesure 2,88 à $3,36 \mathrm{~mm}$ de longueur; celle du protibia est de 1,76 à 2,08 ; celle du protarse varie de 0,56 à 0,72 ; celle du mésofémur est de 2,88 à 3,84 ; celle du mésotibia est de 2,64 à 3,20 ; celle du mésotarse varie de 1,20 à 1,52 ; celle du métafémur varie de 4,16 à 4,72 ; celle du métatibia est de 4,00 à 4,64; celle du métatarse varie de 1,92 à 2,32 . Les adultes sont 1,5 fois plus longue que large, la tête est 2,5 fois plus large que longue. L'hémélytre est environ 2,45 fois plus long que large. Son corie est formé de l'exocorie, de l'endocorie et du clavus qui a une forme triangulaire. 

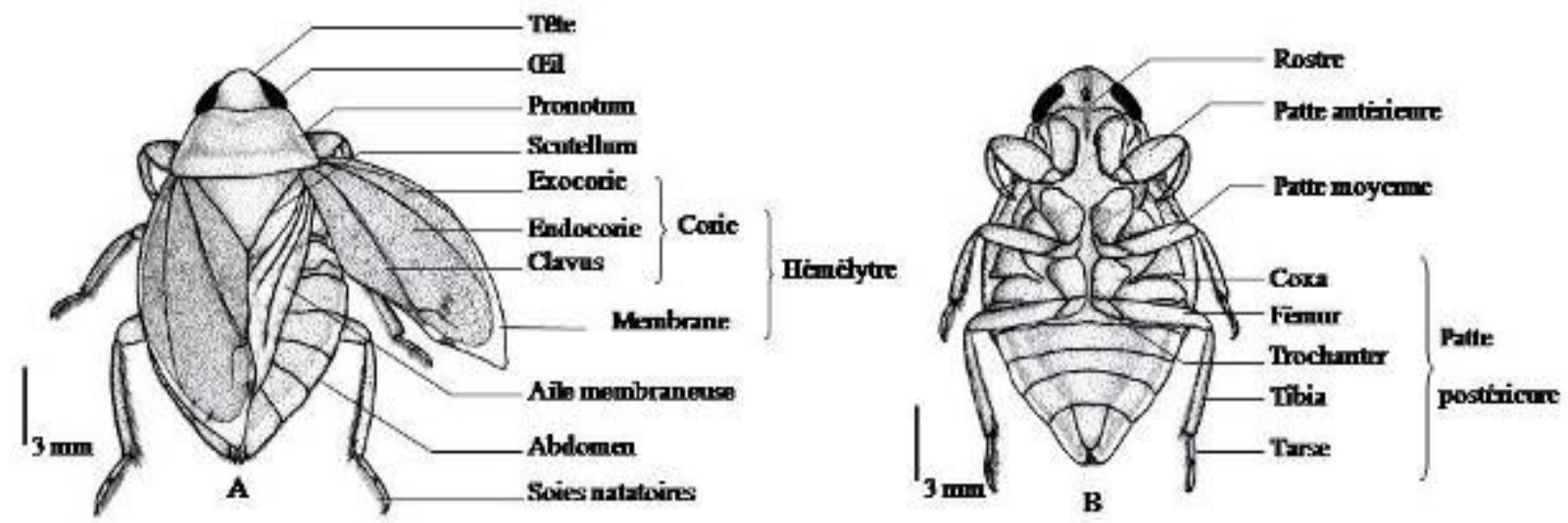

Figure 5: Morphologie d'un llyocoris cimicoïdes adulte. $A=$ vue dorsale, $B=$ vue ventrale

Abondance des stades de développement postembryonnaires d'l. cimicoïdes : L'étude a permis de collecter 1004 œufs et 1347 stades post-ovulaires. Les larves de stades 1, 2, 3, 4 et 5 et les adultes représentent respectivement $29,03 \%, 21,38 \% ; 18,78 \% ; 12,92 \%$; $7,87 \%$ et $10,02 \%$ des effectifs recensés (figure 6 ). Les larves de stade 1 sont les plus abondantes; elles sont 1,36 fois plus abondantes que les larves de stade $2 ; 1,55$ fois plus abondantes que les larves de stade $3 ; 2,25$ fois plus abondantes que les larves de stade
$4 ; 3,69$ fois plus abondantes que les larves de stade 5 et 2,90 fois plus abondantes que les adultes. Pour la période de l'étude, les œufs sont plus abondants pendant la saison des pluies surtout aux mois de mai et de juillet. Pendant la saison sèche, ils pullulent pendant les mois de février et de mars. Les larves sont abondantes pendant la saison sèche et pullulent en décembre pour les larves 3 , en janvier pour les larves 4 et 5 et en février pour les larves 1 et 2.

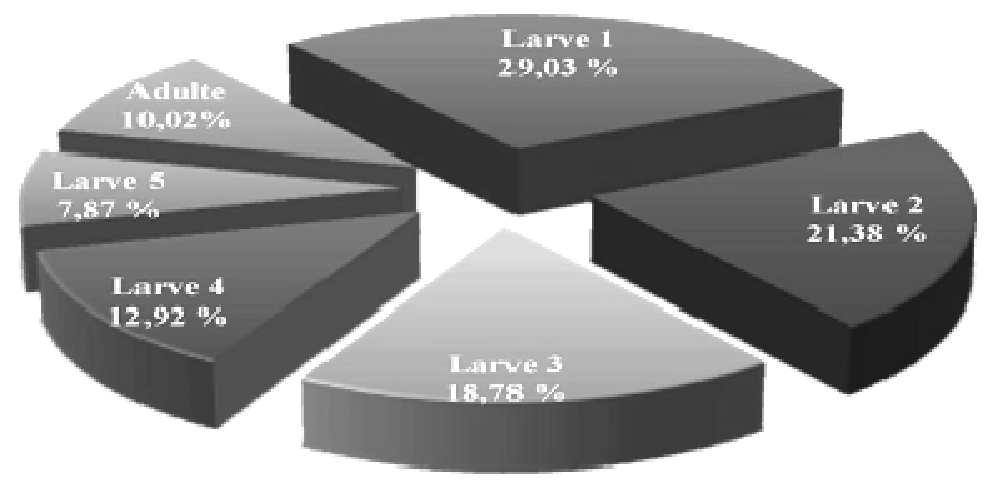

Figure 6 : Abondances relatives des différents stades de développement post-embryonnaire d' llyocoris cimicoïdes récoltés de décembre 2011 à juillet 2012

\section{DISCUSSION}

llyocoris cimicoïdes présente la morphologie externe des insectes hétérométaboles. Les stades larvaires de cet insecte présentent des similarités morphologiques avec celles de Limnocoris lutzi (Heteroptera: Naucoridae) (Sites et Nichols, 2001). Cependant, les larves d'l. cimicoïdes ont deux fois la taille des larves de L. lutzi. En de cette différence, les larves d'l. cimicoïdes n'ont pas de maculation prononcée comme chez celles de L. lutzi. L'étude a également montré que $I$. cimicoïdes se développe dans le lac d'obili (Yaoundé, Cameroun) en six stades parmi lesquels cinq sont larvaires. Ce nombre de stades larvaires d' I. cimicoïdes, est identique à celui noté chez d'autres punaises, notamment Coridius 


\section{Ajeagah et al. J. Appl. Biosci. 2014. Morphologie et abondance des stades de développement}

d'llyocoris cimicoïdes Linne 1758 dans un lac anthropisé en zone tropicale (Cameroun)

xanthopterus Fairmaire 1858 (Fomekong et al, 2009), Sahlbergella singularis Haglund 1895, Distantiella theobromae Distant 1905 et Helopeltis bergrothi Reuter 1892 (Lavabre, 1992). L'évolution temporelle de la dynamique de l'abondance des différents stades de développement d'l. cimicoïdes met en évidence l'effet de la saisonnalité sur le développement de ce Naucoridé. Les conditions qui règnent dans le milieu à chaque saison favorisent l'installation d'un stade donné de développement. En effet, plusieurs facteurs à l'instar du niveau de pollution du milieu, des ressources disponibles, de la présence des prédateurs peuvent avoir une influence sur la fécondité, la longévité, la vitesse de croissance, la mortalité, donc sur le cycle biologique des organismes vivants dans un écosystème (O'Brien et Keough, 2013). A ce titre, l'augmentation de l'abondance des œufs et des adultes observée durant la saison de pluies peut être corrélée à l'augmentation de la quantité de ressources et l'installation des conditions favorables à la maturation sexuelle. En effet, pendant les mois pluvieux, les ruissellements favorisent l'enrichissement du milieu en ressources alimentaires nécessaires à la mue imaginale et à la production d'un nombre important

\section{CONCLUSION}

Au terme de cette étude, l'analyse des variables physicochimiques des eaux du lac d'Obili notamment la transparence et la teneur en orthophosphates montre un état eutrophe à hypereutrophe du lac. L'évolution saisonnière de certaines variables physico-chimiques de la pièce d'eau influence la dynamique de l'abondance des stades de développement post-embryonnaire d'llyocoris cimicoïdes. Les mois pluvieux sont en faveur de l'augmentation du nombre d'œufs pondus et de l'abondance des adultes alors que la sécheresse favorise l'épanouissement des larves. Après l'éclosion des œufs, llyocoris cimicoïdes se développe en six stades postembryonnaires parmi lesquels cinq sont larvaires. Plusieurs caractères morphologiques permettent une

\section{RÉFÉRENCES}

APHA, 1998. Standard method for examination of water and wastewater, American Public Health Association, $20^{\text {th }}$ edition, Washington, DC, 1150 p.

Aubry P. 2011. Ulcère de Buruli, Journal de Médecine tropicale, $5 p$.

Barbour M. T., Gerritsen J., Snyder B. D. et Stribling J. B. 1999. Rapid 999assessment protocols for use in streams and wadeable rivers: periphyton, benthic, macroinvertebrates and fish, second d'œufs d'autant plus que le jeûn chez les insectes conduit à une réduction du nombre d'ovarioles et par conséquent le nombre d'œufs pondus (Dajoz, 2006). Contrairement aux adultes qui s'épanouissent pendant la saison des pluies, les larves ont tendance à être plus nombreux pendant la saison sèche à cause des conditions environnementales défavorables qui règne en saison pluvieuse. En fait, les crues et les ruissellements divers provoquent le plus souvent la destruction des niches et des abris pour les larves et par conséquent leur dérive étant donné que ces insectes colonisent avant tout les eaux privées de courant. Bien que l'effet de la saisonnalité soit remarquable sur le cycle de développement d'I. cimicoïdes, le degré d'eutrophisation semble faire partie des facteurs essentiels à l'installation de ce Naucoridé dans le milieu. Les milieux présentant de fortes teneurs en matières organiques et en nutriments ou milieux soumis à une action anthropique croissante sont propices au développement d'l. cimicoïdes. A ce titre, la présence de ce Naucoridé pourrait être un indicateur des milieux eutrophes à hypereutrophes (Nzieleu,2006 ; Ngoko,2011).

distinction des différents stades larvaires. La larve de 1 er stade se distingue des autres stades larvaires par l'absence du ptérothèque. Les larves de 2 ème et de 3 ème stade ont le bord postérieur médian de leur mésotergum légèrement échancré tandis qu'il est non échancré et arrondi chez les larves de $4{ }^{\text {ème }}$ et $5^{\text {ème }}$ stade. L'extrémité postérieure du ptérothèque a le même alignement que la base médiane du mésotergum chez les larves de 2 ème stade alors qu'elle dépasse légèrement la base du métatergum chez les larves de $3^{\text {ème }}$ et 4 ème stades. Elle atteint dépasse la base du métatergum et atteint le premier segment abdominal visible chez les larves de 5 ème stade.

edition. EPA 841-B-99-002, US Enviromental Protection Agency Office of Water, Washington DC.

Dajoz R. 2006. Précis d'écologie, 8e édition, Dunod, Paris, 630p.

Dethier M. 1980. Hétéroptères. In Durand J. R. et Lévêque $C$., "Flore et faune aquatiques de l'Afrique sahélo-soudanienne » Tome 1, Paris, ORSTOM, 44 : 661-685. 


\section{Ajeagah et al. J. Appl. Biosci. 2014. Morphologie et abondance des stades de développement}

d'llyocoris cimicoïdes Linne 1758 dans un lac anthropisé en zone tropicale (Cameroun)

Ebong S. M., Eyangoh S., Marion E., Landier J., Marsollier L., Guégan J. F. et Legall P. 2012. Survey of water bugs in bankim, a new buruli ulcer endemic area in Cameroon, Journal of Tropical Medicine, 12: 38-43

Fomekong A., Messi J., Kekeunou S., Tamesse J. L. 2009. Développement, morphologie et reproduction de Coridius xanthopterus (Heteroptera: Dinidoridae), ravageur du Concombre Cucumeropsis mannii dans le sudCameroun, Entomologie faunistique, 62 : 153154.

Gamboa M., Kimbirauskas R. K., Merritt R. W. et Monaghan M. T. 2012. A molecular approach to identifying the natural prey of the African creeping water bug Naucoris, a potential reservoir of Mycobacterium ulcerans, Journal Institute of Sciences, 12 (2): 16-31.

Lavabre E.M. 1992. Ravageurs des cultures tropicales, Maisonneuve et Larose et ACCT, Paris, France, $177 p$.

Mohammad S., Nasim J. et Ramesh C. 2011. New record of naucorids (Hemiptera: Heteroptera) in India, Science and Technology, 3(6): 12-15.

Moisan J. et Pelletier L. 2008. Guide de surveillance biologique basée sur les macroinvertébrés benthiques d'eau douce du Québec, Cours d'eau peu profonds à substrat grossier. Direction de suivi de l'état de l'environnement, Ministère du Développement Durable, de l'Environnement et des Parcs, 86p.

Ngoko K. E. 2011. Recherche des oocystes de Cyclospora cayetanensis dans le cours d'eau Olézoa et deux de ses affluents, Mémoire de Master, Faculté des Sciences, Université de Yaoundé I, 54p.

Nzieleu T. J. G. 2006. Étude du déterminisme du polymorphisme des Rotifères Brachionidae dans trois plans d'eau de Yaoundé : Lac Municipal de Yaoundé, étang de Mélen et étang d'Efoulan. Mémoire de DEA, Faculté des Sciences, Université de Yaoundé I, 62p.

O'Brien A. L. et Keough M. J. 2013. Detecting benthic community responses to pollution in estuaries: $A$ field mesocosm approach, Environmental Pollution, 15: 45-55.

OMS, 2004. Ulcère de Buruli. Infection à Mycobacterium ulcerans. REH, 79, 145-149.

Poisson R. 1948. Hydrocorises du Cameroun. Revue Française d'Entomologie, $15:$ 167-177.

Poisson R. 1957. Hétéroptères aquatiques. Faune de France, Lechevalier, Paris, $61: 261 \mathrm{p}$.

Polhemus J. T. et Polhemus D. A. 2008. Global diversity of true bugs (Heteroptera: Insecta) in freswater, Hydrobiologia 595: 379 - 298.

Rodier J. 1996. L'analyse de l'eau: eaux naturelles résiduaires, eau de mer, 8 è ed. Dunod, 1383p.

Ryding S.O. et Rast W. 1994. Le contrôle de l'eutrophisation des lacs et des réservoirs, Masson, Unesco, 294p.

Sites R. W. et Nichols B. J. 2001. Voltinism, egg structure and descriptions of immature stages of Limnocoris lutzi (Heteroptera: Naucoridae). Annal de la Société Americaine d'Entomologie, 94 (1): 26-32.

Sites R. W., Zettel H. et Arunachalam M. 2011. Waterfallinhabiting Naucoridae (Hemiptera : Heteroptera) of southern India and Sri Lanka: Pogonocaudina Sites and Zettel, n. gen., and a review of Diaphorocoris with descriptions of two new species, Zootaxa, 2760: 1- 17.

Tachet H., Richoux P., Bournaud M. et Usseglio-Polatera P. 2002. Invertébrés d'eau douce: systématique, biologie, écologie, Paris, CNRS Ed, 587p. 